\title{
Linear mucosal defects: a characteristic endoscopic finding of lansoprazole-associated collagenous colitis
}

Collagenous colitis is a distinct clinicopathologic entity characterized by chronic watery diarrhea and, histologically, by the subepithelial deposition of collagen $[1,2]$. Some luminal antigens, including nonsteroidal anti-inflammatory drugs (NSAIDs) and proton pump inhibitors (PPIs), particularly lansoprazole, are thought to contribute to the development of collagenous colitis. On endoscopy, collagenous colitis usually shows no or minimal nonspecific abnormalities. However, a recent report showed that "linear mucosal defects", including mucosal tears and sharp longitudinal ulcers, are characteristic colonoscopic findings of lansoprazoleassociated collagenous colitis [3]. We report a case of lansoprazole-associated collagenous colitis that showed multiple linear mucosal defects in the colon.

A 67-year-old woman was referred to our hospital with a 1-month history of continuous watery diarrhea and abdominal pain. The patient had been taking $15 \mathrm{mg}$ of lansoprazole daily for the past 4 months for gastroesophageal reflux disease. She had no previous history of other gastrointestinal disease. The left lower abdomen was painful on palpation. A stool culture was negative for any enteric pathogens. Colonoscopy revealed a linear mucosal defect surrounded by edematous mucosa in the descending colon (๑ Fig. 1) and the proximal sigmoid colon ( Fig. 2). In addition, a linear and scarred mucosal defect was observed in the distal sigmoid colon ( $\bullet$ Fig. 3). The sharply demarcated margin of the mucosal defect differentiated it from ischemic colitis. Multiple biopsy specimens, including those around the mucosal defect, were found to have thickened subepithelial collagen bands and inflammation of the lamina propria ( $\bullet$ Fig.4). This patient's clinical and histological findings

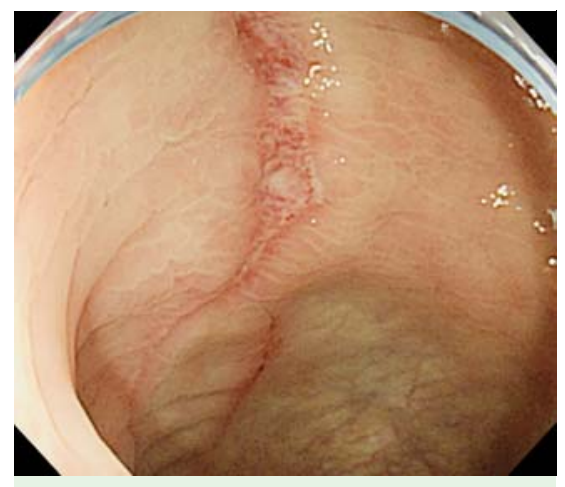

Fig. 1 Colonoscopy showing a linear mucosal defect surrounded by edematous mucosa in the descending colon.

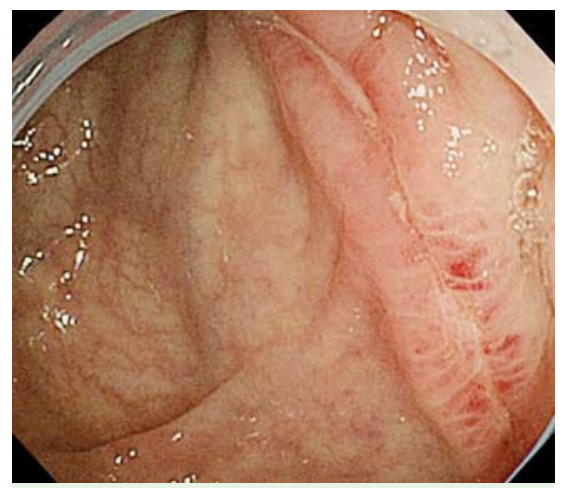

Fig. 2 Colonoscopy showing a linear mucosal defect in the proximal sigmoid colon.

were compatible with the diagnosis of collagenous colitis. After discontinuation of lansoprazole treatment, the patient's symptoms improved.

Although the endoscopic finding in collagenous colitis is generally considered to be normal or near-normal mucosa, observation of a linear mucosal defect is useful for diagnosis of lansoprazole-associated collagenous colitis.

Endoscopy_UCTN_Code_CCL_1AD_2AD
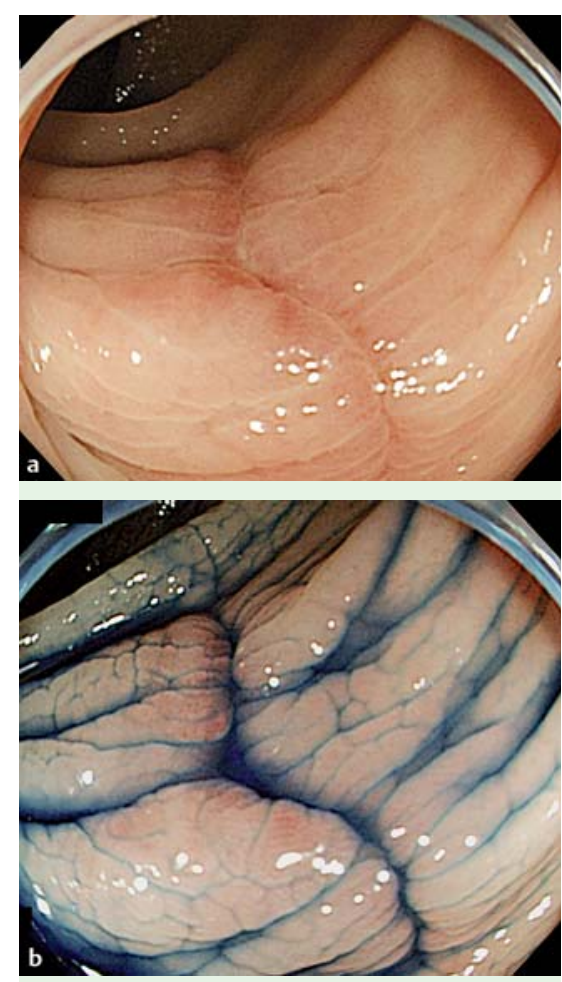

Fig. 3 a Linear and scarred mucosal defect in the distal sigmoid colon. $\mathbf{b}$ Chromoendoscopic view with indigo carmine contrast clearly demonstrating a linear scar.

E. Nomura ${ }^{1}$, H. Kagaya ${ }^{1}$, K. Uchimi ${ }^{1}$,

T. Noguchi ${ }^{1}$, S. Suzuki ${ }^{1}$, M. Suzuki ${ }^{1}$, H. Onodera ${ }^{1}$, H. Tateno ${ }^{2}$

1 Department of Gastroenterology, Miyagi Cancer Center, Miyagi, Japan

2 Department of Pathology, Miyagi Cancer Center, Miyagi, Japan 


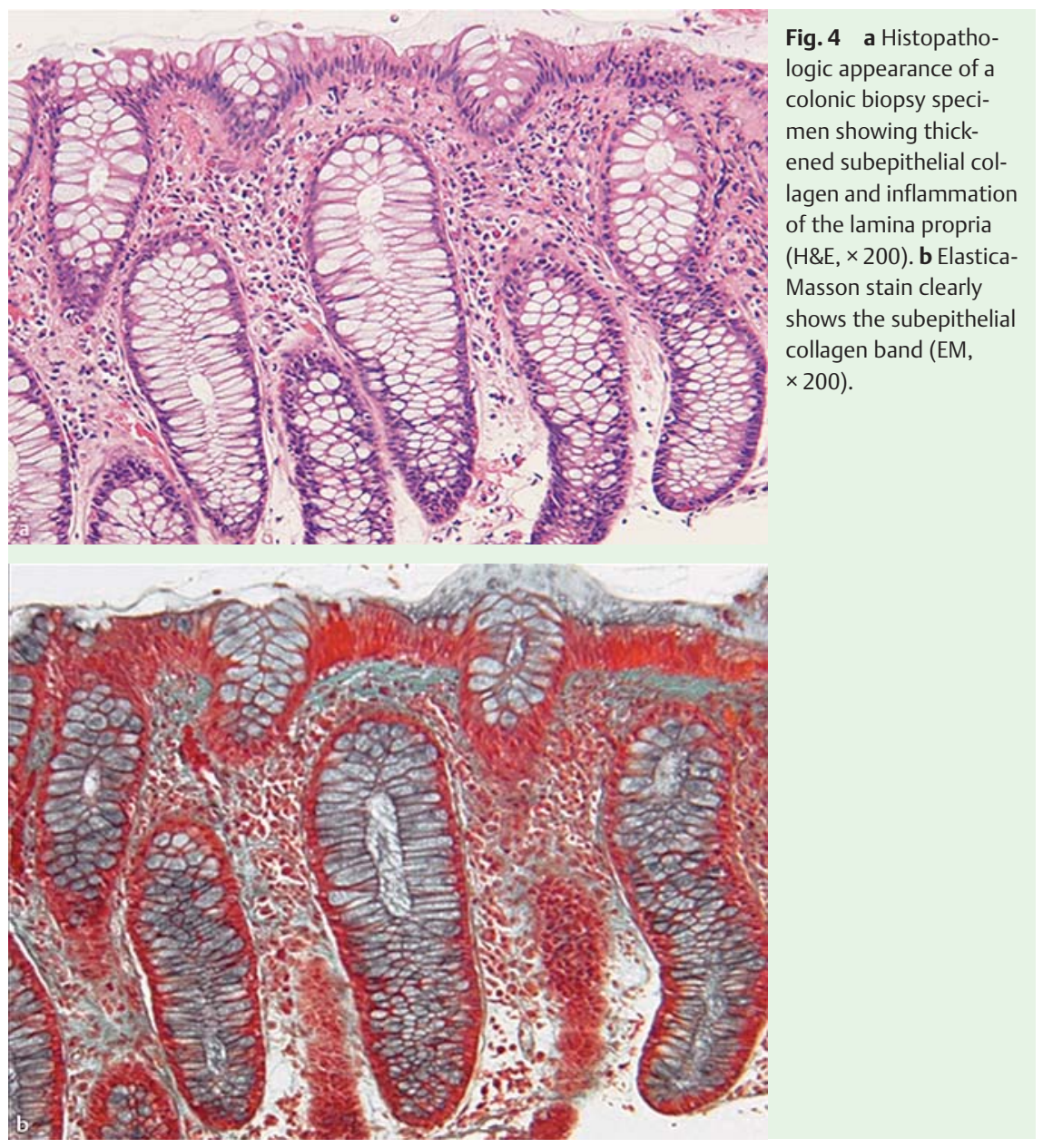

\section{References}

1 Lindström CG. Collagenous colitis with watery diarrhea. A new entity? Pathol Eur 1976; 11: $87-89$

2 Bohr J, Tysk C, Eriksson S et al. Collagenous colitis: a retrospective study of clinical presentation and treatment in 163 patients. Gut 1996; 39: 846 - 851

3 Umeno J, Matsumoto T, Nakamura $S$ et al. Linear mucosal defect may be characteristic of lansoprazole-associated collagenous colitis. Gastrointest Endosc 2008; 67: 11851191

\section{Bibliography}

DOI 10.1055/s-0029-1214795

Endoscopy 2010; 42: E9-E10

(c) Georg Thieme Verlag KG Stuttgart · New York . ISSN 0013-726X

\section{Corresponding author \\ E. Nomura, MD}

47-1 Nodayama

Shiode Medeshima

Natori

Miyagi 981-1293

Japan

Fax: +81-22-381-1168

nom_e@mwd.biglobe.ne.jp 